\section{A Simple Modified Flip-Flop Flap for the Reconstruction of Antihelix and Triangular Fossa Defects}

Min Joon Oh, Seok Joo Kang, Hook Sun

Department of Plastic and Reconstructive Surgery, Busan-Baik Hospital, Inje University School of Medicine, Busan, Korea

\section{Correspondence: Seok Joo Kang}

Department of Plastic and Reconstructive Surgery, Busan Baik Hospital, Inje University School of Medicine, 75 Bokji-ro, Busanjin-gu, Busan 47392, Korea Tel: +82-51-890-6136, Fax: +82-51-894-7976, E-mail: sonydr@naver.com

No potential conflict of interest relevant to this article was reported.

Received: 23 Mar 2015 • Revised: 16 Apr 2015 • Accepted: 23 Apr 2015 pISSN: 2234-6163 • elSSN: 2234-6171

http://dx.doi.org/10.5999/aps.2016.43.1.122

Arch Plast Surg 2016;43:122-124

Copyright (C) 2016 The Korean Society of Plastic and Reconstructive Surgeons

This is an Open Access article distributed under the terms of the Creative Common Attribution Non-Commercial License (http://creativecommons.org/licenses/by-nc/3.0/) which permits unrestricted non-commercial use, distribution, and reproduction in any medium, provided the original work is properly cited.

Due to the three-dimensional configuration of the auricle and cartilage, surgeons encounter significant challenges in the reconstruction of auricular defects in anterior regions such as the antihelix and triangular fossa. Local flaps, skin grafting, and wedge excision are currently used in these locations. Postauricular

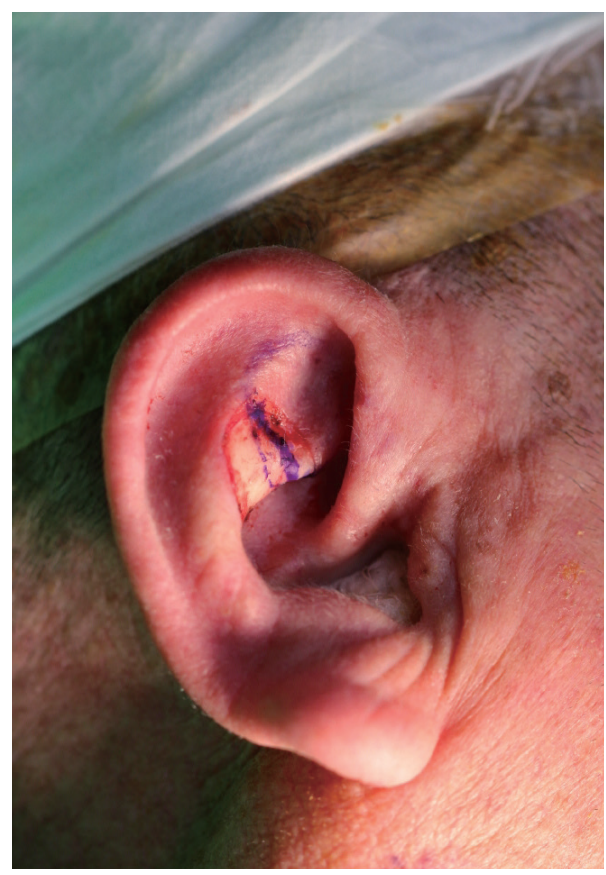

Fig. 1.

The defect, located throughout the antihelix and triangular fossa, was oval in shape and $2.0 \times 1.8 \mathrm{~cm}$ in size, with exposed cartilage. We decided to create a $30 \%$ overlap in the medial direction. flaps are the most common of these techniques, due to their advantages that include low morbidity, a wellhidden scar, direct closure, and good color match to the ear skin [1]. However, the lack of underlying vasculature and perichondrium may lead to graft necrosis. Moreover, wedge excision cannot be performed in patients with defects of the antihelix or triangular fossa because such patients are vulnerable to severe ear deformities.

We modified a flip-flop flap to reconstruct anterior defects of the ear across the antihelix and triangular fossa. Our technique is a one-stage, random-pattern island flap surgery, and allows a shorter operation time and reduced losses at the donor site, while still leading to satisfactory treatment outcomes. Here, we describe our technique and its outcome. This study was reviewed and approved by the ethics review board of the Inje University Health Center.

A 70-year-old man visited us with a chief complaint of Bowen's disease in the right ear. The patient underwent excision of the tumor with adequate circumferential and deep margins, including the perichondrium. The defect, located throughout the antihelix and triangular fossa, was oval in shape and $2.0 \times 1.8 \mathrm{~cm}$ in size, with exposed cartilage (Fig. 1).

Under local anesthesia, we performed preoperative marking for the skin island, based on the size of the

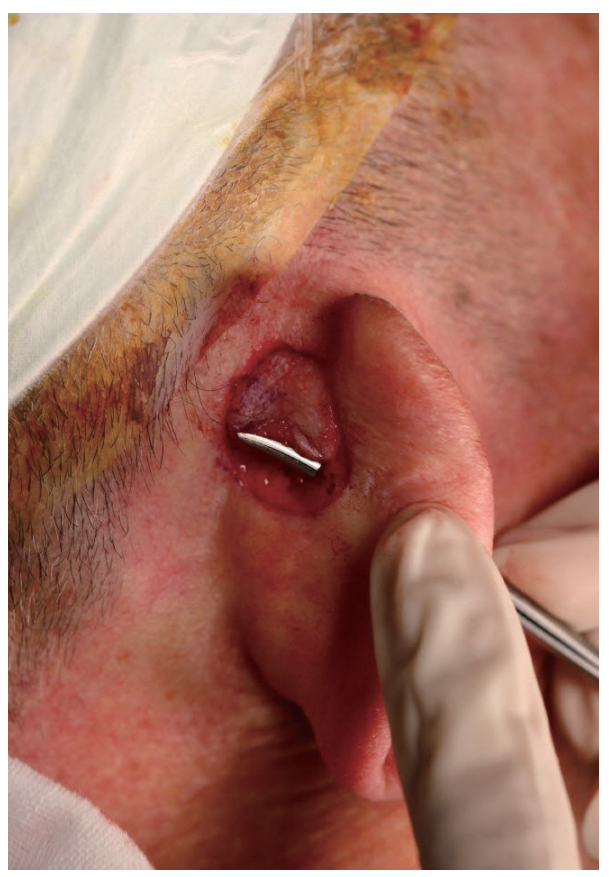

Fig. 2.

After creating a skin island, we removed a small, slender piece of cartilage to provide an entrance for the skin flap. 


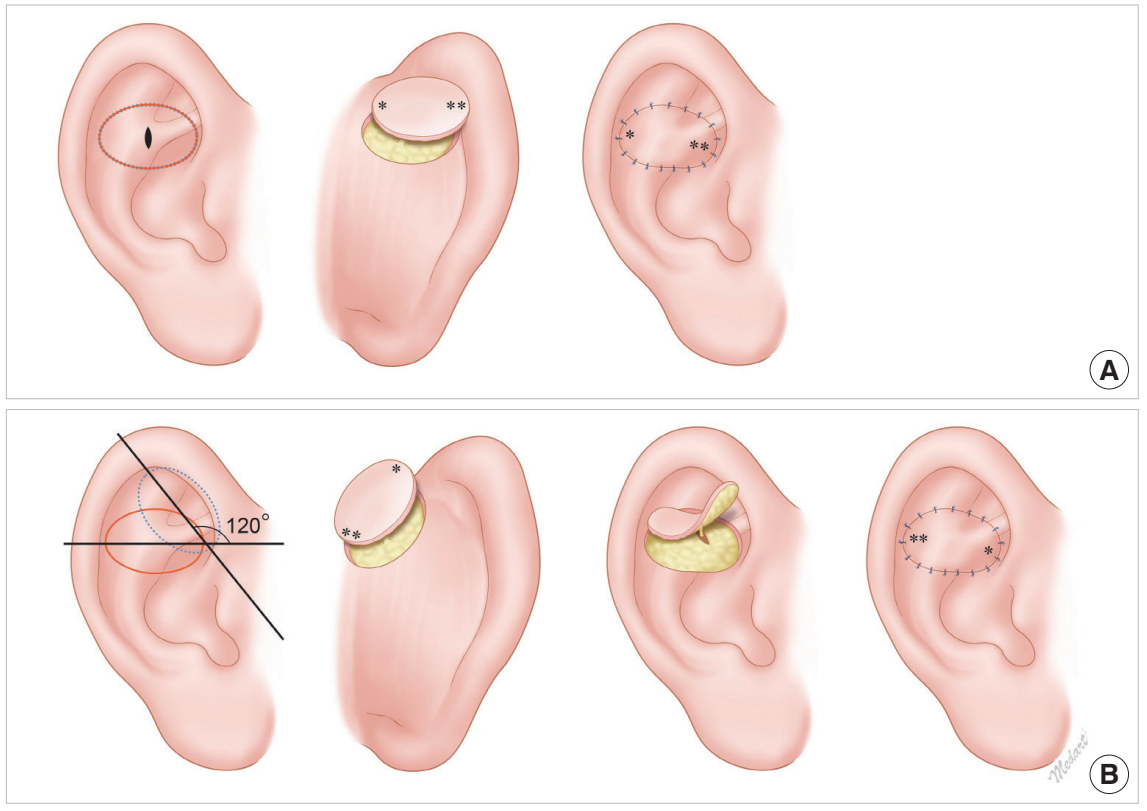

Fig. 3.

Schematic diagram. (A) Schematic illustration of a conventional flip-flop flap. There is an exact match in surgical design between the anterior defect site and the posterior donor site. The lateral side ${ }^{* *}$ ) of the donor site exits anteriorly through the cartilage window and then is rotated at an angle of $180^{\circ}$. Thus, it reaches the medial side ${ }^{* *}$ of the anterior defect site. (B) Schematic illustration of a modified flip-flop flap. A $120^{\circ}$ angle is formed between the long axis of the donor site (dotted line) and that of the defect (line). The lateral side of the donor site $\left(^{*}\right)$ is rotated at an angle of $120^{\circ}$ through the cartilage window and then reaches the medial side of the ear $\left(^{*}\right)$. anterior auricular defect, for which we intended a $30 \%$ overlap in the medial direction (Fig. 1). A cartilage window was then drawn at the center of the overlap between the marked area and the actual defect. Thus, we attempted to provide an entrance for skin flap placement (Fig. 2).

After being drawn on the anterior auricular surface, the pattern was marked on the back and then redrawn. It was then redesigned by extending the top side by approximately $10 \mathrm{~mm}$, in line with the sulcus axis, thus leaving the lower base intact. In other words, it was shaped into an elongated orb alongside the axis of the sulcus. We thus made it easier to reduce the tension in the central pedicle during rotation and to suture the donor site. We placed one third of the skin island over the mastoid area and the remaining two thirds of the skin island over the postauricular surface.

After excising the designated area, we elevated the skin flap while preserving the central subcutaneous pedicle. Once the flap was observed to be sufficiently elevated, we rotated it at an angle of $120^{\circ}$ around the long axis through the cartilage window (Fig. 3). The flap was closed using interrupted prolene 6-0 sutures, and the postauricular donor site was closed using continuous vicryl 5-0 and prolene 5-0 sutures.

The donor site was easily closed through direct repair, without other procedures. No differences were observed in the color and thickness of the skin between the flap and the anterior auricle. Moreover, the patient did not develop postoperative infections or flap loss. Furthermore, at a follow-up, the patient had no ear asymmetry or other complications, such as notable scarring (Fig. 4).

In 1972, Masson [2] first reconstructed anterior auricular defects using subcutaneous pedicle postauricular flaps. These flaps have also been referred to as revolving or flip-flop flaps, or as posterior auricular rotation flaps, and they have been reported to be appropriate for reconstructing defects on the scapha, triangular fossa, antihelix, and conchal bowl. The advantages of this technique include the availability of well-vascularized skin for the coverage of large auricular defects and a one-stage operative procedure [3]. Since first being introduced, Masson's flap has been used by many surgeons. Due to the short length and immobilization of the subcutaneous pedicle, however, it has limitations in lowering the ear projection and the depth of the sulcus.

In order to increase flap mobility, some authors have described postauricular axial pattern flaps, in which the posterior auricular artery or the superior auricular artery is used as a pedicle [4]. However, these flaps are disadvantageous in that they require careful dissection and may result in morbidity. Therefore, because they are not used frequently, other researchers have developed a de-epithelialized postauricular flap; its advantages include higher safety and mobility compared to the subcutaneous pedicle flap and lower morbidity than the axial flap.

Nonetheless, it is problematic that additional procedures are required for de-epithelialization, and ear deformities may occur as a result of the increased area of the postauricular donor site. 


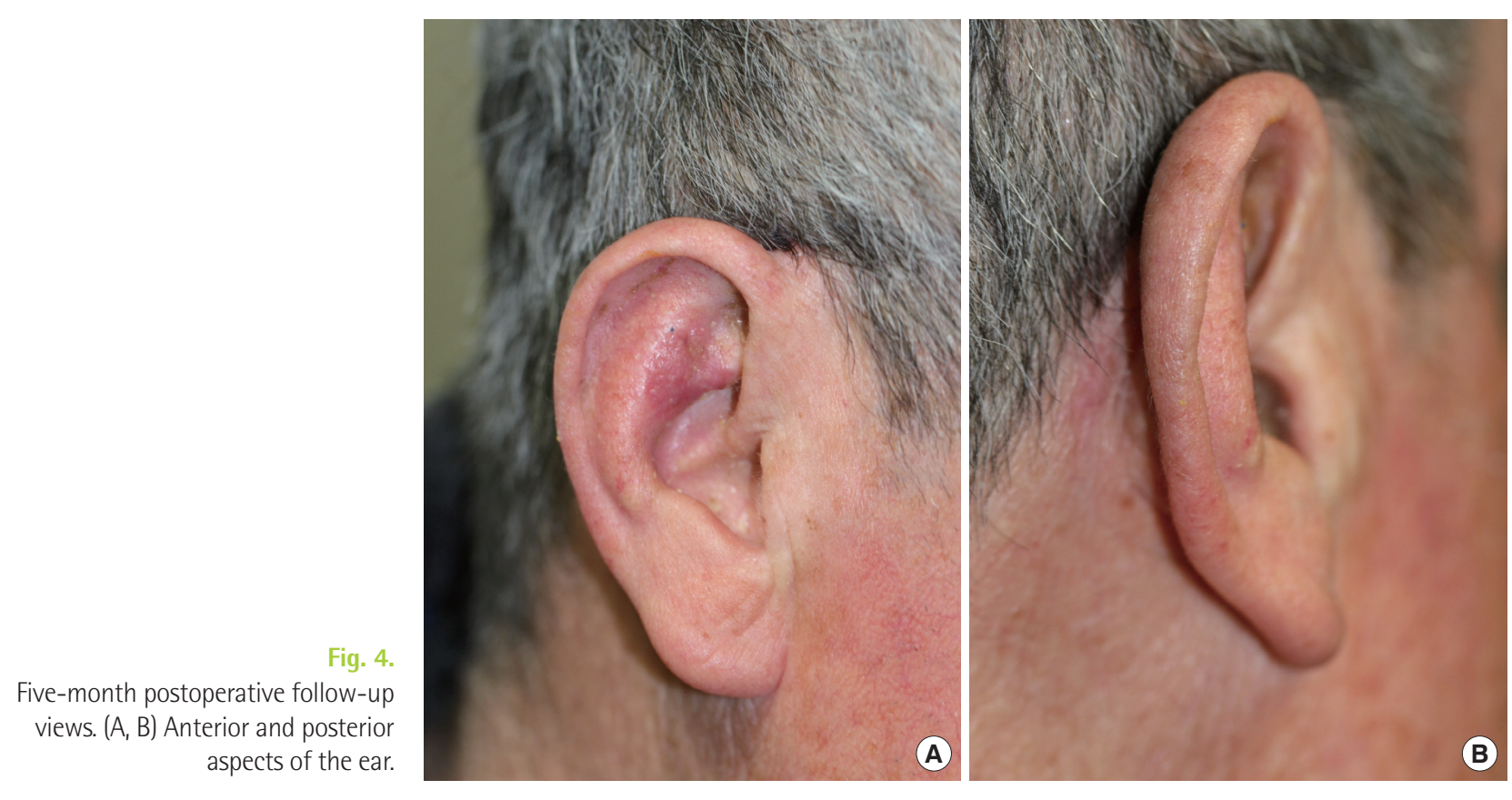

Our technique can be summarized as follows. First, we approached the postauricular donor site proximally to the sulcus in the medial direction rather than in same region as the defect. Thus, we were able to obtain a sufficient amount of postauricular skin, even in patients with defects of the outer edge of the ear, such as the antihelix or scapha.

Second, we rotated the postauricular skin island at an angle of $120^{\circ}$, unlike conventional methods in which it is rotated at an angle of $180^{\circ}$ through the cartilage [5]. This is useful when there is no parallel relationship between the axis of the largest defects and that of the sulcus or when the angle is approximately $90^{\circ}$. Aligning the maximum width of the defect to the postauricular sulcus axis, which has sufficient space, reduced the size of the defect in the donor region while simultaneously allowing a safe blood supply.

Third, we did not create the cartilage window at the center of the flap island, but instead made it as small as possible in the overlapping region between the defect and the donor site. Thus, we increased the stability of the skin flap by preventing the pedicle from being entangled.

We modified a postauricular island flap to reconstruct defects across the antihelix and triangular fossa. Our technique was useful not only in maintaining the contour and three-dimensional structure of the auricle, but also in shortening the operation time and recovery period.

\section{References}

1. Larcher L, Plotzeneder I, Tasch C, et al. Retroauricular pull-through island flap for defect closure of auricular scapha defects: a safe one-stage technique. J Plast Reconstr Aesthet Surg 2011;64:934-6.

2. Masson JK. A simple island flap for reconstruction of concha-helix defects. Br J Plast Surg 1972;25:399-403.

3. Schonauer F, Vuppalapati G, Marlino S, et al. Versatility of the posterior auricular flap in partial ear reconstruction. Plast Reconstr Surg 2010;126:1213-21.

4. Turan A, Turkaslan T, Kul Z, et al. Reconstruction of the anterior surface of the ear using a postauricular pull-through neurovascular island flap. Ann Plast Surg 2006;56:609-13.

5. Talmi YP, Wolf M, Horowitz Z, et al. "Second look" at auricular reconstruction with a postauricular island flap: "flip-flop flap". Plast Reconstr Surg 2002;109:713-5. 